\title{
The Role of Colostrum Feeding on the Nutritional Status of Preschool Children in Afambo District, Northeast Ethiopia: Descriptive Cross Sectional Study
}

\author{
Misgan Legesse Liben ${ }^{1, *}$, Taye Abuhay ${ }^{2}$, Yohannes Haile ${ }^{3}$ \\ ${ }^{1}$ Department of Public Health, College of Medical and Health Sciences, Samara University, Afar, Ethiopia \\ ${ }^{2}$ Department of Statistics, Institute of Technology, Bahir Dar University, Amhara, Ethiopia \\ ${ }^{3}$ Department of Statistics, College of Natural and Computational Science, Samara University, Afar, Ethiopia \\ Email address: \\ lmisgan@yahoo.com (M. L. Liben) \\ ${ }^{*}$ Corresponding author
}

To cite this article:

Misgan Legesse Liben, Taye Abuhay, Yohannes Haile. The Role of Colostrum Feeding on the Nutritional Status of Preschool Children in Afambo District, Northeast Ethiopia: Descriptive Cross Sectional Study. European Journal of Clinical and Biomedical Sciences.

Vol. 2, No. 6, 2016, pp. 87-91. doi: 10.11648/j.ejcbs.20160206.15

Received: September 9, 2016; Accepted: November 28, 2016; Published: December 27, 2016

\begin{abstract}
Abstarct: Introduction: The World Health Organization (WHO) and United Nations Children's Emergency Fund (UNICEF) recommend colostrum feeding in the first few days after birth. Colostrum is the first milk containing proteins, vitamin A and maternal antibodies. Objective: This study was aimed to assess the role of colostrum feeding on the nutritional status of preschool children in Afambo district, Afar Region, Northeastern Ethiopia. Methods: A community based cross sectional study was conducted in Afambo district of Afar Regional State from January 21 to February 05/2015. Anthropometric measurements (weight and length/height) were done for all study children. WHO Anthro version 3.2.2 was used to convert height and weight measures into z-scores using the WHO reference population. Then, Chi-square test statistic was used to test the association between colostrum feeding and child under nutrition. Results: A total of 370 mother-child pairs were included in the study. Ninety one percent of the study mothers had ever breastfed their index child. About $35 \%$ of the mothers discarded colostrums. The chi-square test showed that colostrum feeding was associated with the three indicators of child under nutrition (stunting, underweight and wasting). Conclusion: This study showed that colostrum feeding is associated with the lower probabilities of under nutrition among preschool children. Therefore, all health partners aimed at improving the nutritional status of preschool children should give emphasis on the importance of colostrum feeding in the study area.
\end{abstract}

Keywords: Afambo, Colostrum, Ethiopia, Nutritional Status, Preschool Children

\section{Introduction}

The World Health Organization (WHO) and United Nation Children's Emergency Fund (UNICEF) recommended exclusive breastfeeding for children up to six months of age, and to nourish them with appropriate complementary foods and continued breastfeeding until 24 months or beyond [1]. Ethiopia also adopted this recommendation $[2,3]$. Therefore, to enable mothers to establish and sustain exclusive breastfeeding for the first six months, it is recommended to practice early initiation of breastfeeding, colostrum feeding, exclusive breastfeeding, on-demand breastfeeding, and no use of bottles, teats or pacifiers [1].
Colostrum is the first liquid which is thick, sticky, and clear to yellowish in color that contains proteins, vitamin A and maternal antibodies. Therefore, it is considered as baby's first immunization [1, 4]. Furthermore, it has a positive effect in the prevention of childhood malnutrition [5]. In spite of this fact, colostrum is discarded as unclean and bad for the infant's health [6-10].

Optimal breastfeeding is associated with lower risk of child morbidity and mortality in the first two years of life. Based on the Breastfeeding Performance Index, only 23.6\% of Ethiopian infants had received optimal breastfeeding. Optimal breastfeeding is associated with lower risk of illness in infants aged less than six months [11]. Suboptimal breastfeeding results in more than 800,000 child deaths 
annually worldwide [12]. Of these, $22.3 \%$ of the neonatal deaths could be prevented if all children had been breastfed in the first hour of life [13].

Child nutritional status is primarily measured by child's growth in height and weight. Children below minus two standard deviation (-2SD) from the median of the WHO reference population for weight-for-age, length/height-forage and weight-for-length/height are considered as underweight, stunted and wasted, respectively [4].

Although colostrum feeding is recommended in the Ethiopian Infant and Young Child Feeding Guideline [2], it is discarded in many parts of the country including Afar Regional State. Therefore, this study was aimed to assess the role of colostrum feeding on the nutritional status of preschool children in Afambo district of Northeastern Ethiopia. The findings of this study will be vital for health service providers, policy makers and program managers to design intervention strategies in promotion of optimal breastfeeding practices and childhood nutritional status in the study area and Afar Regional State as a whole.

\section{Methods}

\subsection{Study Setting and Design}

A community based cross sectional study was conducted in Afambo district of Afar Regional State from January 21 to February 05/2015. There are seven kebeles (the smallest administrative units next to district in Ethiopia). Based on the 2007 census population projection, the district has a total population of 29,399, of which 2,639 are estimated to be children aged 6-59 months.

\subsection{Sampling Procedure and Technique}

A total of 370 mother-child pairs were included in this study. First, Afambo district was selected purposively. Secondly, from the seven kebeles four were randomly selected by lottery. To give equal chance in the selection of the sampling unit, proportional allocation technique was employed across each selected kebeles. Then, to select the sampling units (households having children aged 6-59 months), first the total number of households with children aged 6-59 months was divided by the sample size in each selected kebeles and then systematic random sampling technique was applied.

At the time of survey, from each household unit one eligible mother-child pair was selected. If there was more than one mother-child pair in one household unit, one mother with the youngest child was selected. From mothers who had more than one child aged 6-59 months, the youngest child was selected as the reference.

\subsection{Data Collection Process and Instruments}

Data were collected using a pre-tested, interviewer administered structured questionnaire. The questionnaire was prepared first in English and translated in to Afar'af (the local language), then back to English to check for consistency. The Afar'af version of the questionnaire was used to collect the data. Eight high school graduates who can speak the local language were recruited as data collectors. Then, the questionnaire was pretested on two kebeles which were not included in the research. The pretest was done to ensure clarity, wordings, logical sequence and skip patterns of the questions. Finally, the pretest amendments on the questionnaire were made accordingly.

Anthropometric measurements (weight and length/height) were done for all children included in the study. Length of the infants (6-23 months) was measured in a recumbent position using a board with a wooden base and movable headpiece. Height of children older than two years was measured in standing position. Weight was measured in kilogram using digital weight scale which was checked against a standard weight for its accuracy on daily basis. Calibration of instrument against zero reading was made following weighting of every child. Children were weighted with light clothing, without shoes. All anthropometric measurements were taken by high school graduates with an assistant.

\subsection{Study Variables}

The outcome variables were wasting, stunting, underweight. The independent variables were child feeding practices (colostrum feeding, early initiation of breastfeeding, prelacteal feeding and bottle feeding in the 24 hours preceding the survey), maternal and household characteristics.

\subsection{Data Processing and Analysis}

Data were checked for completeness and consistencies. It was also cleaned, coded and entered into Epi Data version 3.02. WHO Anthro version 3.2.2 was used to convert height and weight measures into $\mathrm{z}$-scores using the WHO reference population.

Then, SPSS version 20 was used to analyze the data. Descriptive statistics were used to compute means and standard deviations for numerical variables as well as frequencies for nominal and ordinal variables. The Chi square test statistic was used to test the association between colostrum feeding and child under nutrition. A value of $\mathrm{P}<0.05$ was considered statistically significant.

\subsection{Ethical Considerations}

The study was approved by the Ethical Review Committee (ERC) of Samara University. An official letter was written from Samara University to the Afambo district administration office. Then, permission and support letters were written to the selected kebeles. The participants enrolled in the study were informed about the study objectives, expected outcomes, benefits and the associated risks. A written consent was taken from the mothers before the interview. Confidentiality of responses was maintained throughout the study.

\section{Results}

\subsection{Characteristics of the Study Subjects}

A total of 370 mother-child pairs were included in the study. 
About $84 \%$ of the mothers were Afar by ethnicity. Nearly $68 \%$ of the mothers did not attend formal education (Table 1).

Table 1. Characteristics of the study subjects in Afambo district of Afar Regional State, Northeast Ethiopia, 2015.

\begin{tabular}{|c|c|c|}
\hline Variables & Frequency & Percent (\%) \\
\hline \multicolumn{3}{|l|}{ Mother's age (year) $(n=370)$} \\
\hline$<20$ & 19 & 5.1 \\
\hline $20-34$ & 265 & 71.6 \\
\hline$>34$ & 86 & 23.3 \\
\hline Mean $( \pm$ SD $)$ age of mothers & $29.16( \pm 6.20$ & \\
\hline \multicolumn{3}{|l|}{ Ethnicity $(n=370)$} \\
\hline Afar & 312 & 84.3 \\
\hline Amhara & 55 & 14.9 \\
\hline Tigray & 3 & 0.8 \\
\hline \multicolumn{3}{|l|}{ Religion $(n=370)$} \\
\hline Muslim & 314 & 84.9 \\
\hline Ethiopian Orthodox & 56 & 15.1 \\
\hline \multicolumn{3}{|l|}{ Mother's occupation $(n=370)$} \\
\hline Housewife & 224 & 60.5 \\
\hline Agro-pastoralist & 115 & 31.1 \\
\hline Government employee & 20 & 5.4 \\
\hline Trader & 11 & 3.0 \\
\hline \multicolumn{3}{|l|}{ Mother's education status $(n=370)$} \\
\hline No formal education & 253 & 68.4 \\
\hline Formal education & 117 & 31.6 \\
\hline \multicolumn{3}{|l|}{ Mother's marital status $(n=370)$} \\
\hline Currently unmarried* & 21 & 5.7 \\
\hline Married & 349 & 94.3 \\
\hline \multicolumn{3}{|l|}{ Household head $(n=370)$} \\
\hline Mothers of the index children & 133 & 35.9 \\
\hline Fathers of the index children & 237 & 64.1 \\
\hline \multicolumn{3}{|l|}{ Child's sex $(n=370)$} \\
\hline Male & 178 & 48.1 \\
\hline Female & 192 & 51.9 \\
\hline \multicolumn{3}{|l|}{ Child's age (in month) $(\mathrm{n}=370)$} \\
\hline $6-17$ & 146 & 39.5 \\
\hline $18-29$ & 126 & 34.0 \\
\hline $30-41$ & 68 & 18.4 \\
\hline $42-53$ & 23 & 6.2 \\
\hline$\geq 54$ & 7 & 1.9 \\
\hline Mean $( \pm \mathrm{SD})$ age of children & $22.42( \pm 12.6$ & \\
\hline
\end{tabular}

*Single, widowed, divorced. $\mathrm{SD}=$ standard deviation.

\subsection{Child Feeding Practices}

Ninety one percent of the study mothers had ever breastfed their index child. About 35\% of the mothers discarded colostrums. Of the mothers who had ever breastfed their index child, 49.6\% reported giving prelacteal feeds to their children (Table 2).

Table 2. Breastfeeding practices in Afambo district of Afar Regional state, Northeast Ethiopia, 2015.

\begin{tabular}{|c|c|c|}
\hline Variables & Frequency (n) & Percent (\%) \\
\hline \multicolumn{3}{|c|}{ Ever breastfeeding $(\mathrm{n}=370)$} \\
\hline Yes & 337 & 91.1 \\
\hline No & 33 & 8.9 \\
\hline \multicolumn{3}{|c|}{ Early breastfeeding $(\mathrm{n}=337)$} \\
\hline Yes & 201 & 40.4 \\
\hline No & 136 & 59.6 \\
\hline \multicolumn{3}{|c|}{ Prelacteal feeding $(n=337)$} \\
\hline Yes & 167 & 49.6 \\
\hline No & 170 & 50.4 \\
\hline \multicolumn{3}{|c|}{ Colostrum feeding $(n=370)$} \\
\hline Yes & 241 & 65.1 \\
\hline No & 129 & 34.9 \\
\hline \multicolumn{3}{|c|}{ Bottle feeding in the previous 24 hours $(n=370)$} \\
\hline Yes & 98 & 26.5 \\
\hline No & 272 & 73.5 \\
\hline
\end{tabular}




\subsection{Chi-square Test Results}

The chi-square test showed that colostrum feeding was associated with the three indicators of child under nutrition (stunting, underweight and wasting) (Table 3).

Table 3. Chi-square test result of child under nutrition by colostrum feeding in Afambo district of Afar Regional state, Northeast Ethiopia, 2015.

\begin{tabular}{|c|c|c|c|c|c|c|c|c|c|}
\hline \multirow{2}{*}{ Variables } & \multicolumn{3}{|c|}{ Stunting } & \multicolumn{3}{|c|}{ Underweight } & \multicolumn{3}{|c|}{ Wasting } \\
\hline & Yes & No & P-Value & Yes & No & P-Value & Yes & No & P-Value \\
\hline \multicolumn{10}{|c|}{ Colostrum feeding } \\
\hline No & 51 & 78 & \multirow{2}{*}{$0.035^{*}$} & 44 & 85 & \multirow{2}{*}{$0.001^{*}$} & 27 & 102 & \multirow{2}{*}{$0.006 *$} \\
\hline Yes & 68 & 173 & & 43 & 198 & & 24 & 217 & \\
\hline
\end{tabular}

* Statistically significant.

\section{Discussion}

Optimal breastfeeding practices are essential to meet the nutritional needs of children in the first few years of life [2]. This study was aimed to assess the role of colostrum feeding on child nutritional status. The prevalence of colostrum feeding in Afambo district was $65.1 \%$. This is lower than the findings at Jimma Arjo district [7], Raya Kobo district [8], Goba district [14] and Dubti town [15]. This difference could be due to the difference in infant feeding styles and sociocultural practices across the communities.

The chi-square test showed that colostrum feeding was significantly associated with stunting, wasting and underweight. In West Gojjam, the main contributing factor for stunting among under five children was deprivation of colostrum [5]. In Somali Region of Ethiopia, proper breastfeeding practices were significantly associated with lower odds of wasting [16]. Similar finding was reported at Nigeria [17]. This could be explained in such a way that colostrum contains nutrients and antibodies that are vital in the prevention of infections and nutritional problems.

This study could have the following limitations. The findings could be affected by recall, interviewer, social desirability and anthropometric measurement bias. However, due attention was given to the training of data collectors, standardization of anthropometric measurements and close supervision throughout the field activities.

\section{Conclusion}

This study showed that colostrum feeding is associated with lower probabilities of child under nutrition. This has implications for health service providers, policy makers and program managers in designing intervention strategies aimed at improving nutritional status of preschool children. Therefore, governmental and nongovernmental health partners in the study area need to emphasize and support the importance of colostrum feeding.

\section{Funding}

Samara University (funder) had no role in the study design, data collection and analysis, decision to publish, or preparation of the manuscript.

\section{Authors' Contributions}

All authors conceived, designed the study, and supervised the data collection. MLL performed the data analysis, interpretation of data and drafted the manuscript. All authors had read and approved the final manuscript.

\section{Acknowledgments}

Authors thank Samara University for supporting this study. Authors also thank supervisors, data collectors and the study subjects.

\section{References}

[1] WHO/UNICEF: Baby-friendly hospital initiative (BFHI). Revised, updated and expanded for integrated care. Section 3, Breastfeeding promotion and support in a baby-friendly hospital: A 20-hour Course for Maternity Staff. 2009.

[2] Federal Ministry of Health (FMOH). (2004) National strategy for Infant and Young Child Feeding (IYCF). Addis Ababa. Ethiopia.

[3] Federal Ministry of Health. (2005) Family health department. National strategy for child survival in Ethiopia. Addis Ababa. Ethiopia.

[4] Central Statistical Agency [Ethiopia] and ICF International. (2012) Ethiopia Demographic and Health Survey 2011. Addis Ababa, Ethiopia and Calverton, Maryland, USA: Central Statistical Agency and ICF International.

[5] Teshome B, Kogi - Makau W, Getahun Z and Taye G. (2009) Magnitude and determinants of stunting in children under-five years of age in food surplus region of Ethiopia: The case of West Gojjam Zone. Ethiopian Journal of Health Development. 23 (2): 98106.

[6] Laroia N, Sharma D. (2006) The religious and cultural bases for breastfeeding practices among the Hindus. Breastfeed Med. 1: 94-98.

[7] Tamiru D, Belachew T, Loha E and Mohammed S. (2012) Sub-optimal breastfeeding of infants during the first six months and associated factors in rural communities of Jimma Arjo Wor eda, Southwest Ethiopia. BioMed Central. 12:363. 
[8] Legesse M, Demena M, Mesfin F and Haile D. (2015) Factors Associated with Colostrum Avoidance among Mothers of Children Aged less than 24 Months in Raya Kobo district, North-eastern Ethiopia: Community-based Cross-sectional study. Journal of Tropical Pediatrics. 0: 1-7.

[9] Rogers N, Abdi J, Moore D, Nd'iangui S, Smith L, Carlson A, Carlson D. (2011) Colostrum avoidance, prelacteal feeding and late breast-feeding initiation in rural Northern Ethiopia. Public Health Nutr. 14: 2029-36.

[10] Liben ML and Yesuf EM. (2016) Determinants of early initiation of breastfeeding in Amibara district, Northeastern Ethiopia: a community based cross-sectional study. International Breastfeeding Journal. 11:7.

[11] Haile D and Biadgilign S. (2015) Higher breastfeeding performance index is associated with lower risk of illness in infants under six months in Ethiopia. International Breastfeeding Journal. 10:32.

[12] Black RE, Victora CG, Walker SP, Bhutta ZA, Christian P, Onis M, Ezzati M, McGregor SG, Katz J, Martorell R, Uauy R and the Maternal and Child Nutrition Study Group. (2013) Maternal and child under nutrition and overweight in lowincome and middle-income countries. The Lancet. 382: 42751.
[13] Edmond KM, Zandoh C, Quigley MA, Amenga-Etego S, Owusu-Agyei S, Kirkwood BR. (2006) Delayed breastfeeding initiation increases risk of neonatal mortality. J Am Acad Pediatr. 117 (3): E380-386.

[14] Setegn T, Gerbaba M and Belachew T. (2011) Determinants of timely initiation of breastfeeding among mothers in Goba Woreda, South East Ethiopia: A cross sectional study. BMC Public Health. 11:217.

[15] Liben ML, Gemechu YB, Adugnew M, Asrade A, Adamie B, Gebremedin E and Melak Y. (2016) Factors associated with exclusive breastfeeding practices among mothers in Dubti town of Afar regional state, Northeast Ethiopia. International Breastfeeding Journal. 11:4.

[16] Fekadu Y, Mesfin A, Haile D and Stoecker B. J. (2015) Factors associated with nutritional status of infants and young children in Somali Region, Ethiopia: a cross- sectional study. BMC Public Health. 15:846.

[17] Candyce H., Joseph E., Khalid O., Ndem A., Yahaya Y., Anne C., Neil A. (2015) Childhood malnutrition is associated with maternal care during pregnancy and childbirth: a crosssectional study in Bauchi and Cross River States, Nigeria. Journal of Public Health Research. 4 (408): 58-64. 